\title{
Vitrification of In Vitro Produced Porcine Blastocysts: Influence of Cryoprotectants Toxicity and Embryo Age
}

\author{
Lain Uriel Ohlweiler, Joana Claudia Mezzalira \& Alceu Mezzalira
}

\begin{abstract}
Background: Porcine embryos are sensible to all assisted reproduction manipulations, especially the ones that involve cryopreservation. Despite the high cryoprotectant concentrations routinely applied, vitrification is the most effective technique to date. These substances toxicity can also play a negative role in embryo viability. During in vitro porcine embryo production, the speed of development is often unevenly distributed. It is possible that their development speed, affects embryo tolerance to cryoprotectants. This study aimed to evaluate the toxicity of porcine embryos of days 5 or 6 of culture to cryoprotectant agents; as well as to assess embryo survival to vitrification.

Materials, Methods \& Results: Parthenogenetic porcine blastocysts and expanded blastocysts of days 5 and 6 of culture were exposed to toxicity tests (experiments 1 and 2) and vitrification (experiment 3 ) using different protocols. In the first experiment, three different cryoprotectants were used (Dimethyl sulfoxide - DMSO, Ethylene glycol - EG, and Sucrose - SUC), combined in three different associations (G1: $15 \%$ EG + 15\% DMSO with 0.5 M SUC; G2: $16 \%$ EG + 16\% DMSO with 0.4 M SUC; G3: $18 \%$ EG + 18\% DMSO with 0.5 M SUC). In the fresh Control, embryos of day 6 are more sensible than the ones of day 5, whom showed a lower hatching rate (39.7 vs. $60.8 \%$ ). After the toxicity (Experiment 1 ) test, the G1 showed better expansion rates in day 6 (50.0 vs 31.0 and 3.6\% for G2 and G3) and higher hatching of day 6 compared to G2 and G3 (23.2, vs. 8.6 and $0.0 \%$ for G2 and G3). The fresh non hatched embryos at day 8, derived at day 6, had a lower percentage of cells with cleaved caspase-3 (20.2\%) compared with the G1 (30.5\%), G2 (31.4\%) and G3 (30.5\%). The hatched embryos of day 5 from G2 had lower total cell number (TCN) compared with the day 6 hatched embryos, whereas in G1 the TCN was not affected. The second experiment compared EG combined to one of these three extracellular cryoprotectants: Polyvinylpyrrolidone/sucrose/trehalose (respectively groups: PVP, SUC, TRE). The group SUC has raised the best results for day 5 embryos, whereas for day 6 embryos SUC and TRE were both best. The third experiment tested four vitrification protocols, being P1: EG+DMSO+TRE/warming with SUC; P2: EG+DMSO+TRE/ warming TRE; P3: EG+TRE/ warming SUC; P4: EG+TRE/warming TRE. The expansion of vitrified day 5 embryos was higher in the P1 (20.0\%) in comparison with the other three groups (4.3, 4.3 and 4.4\% for P2, P3 and P4, respectively), with no difference for their hatching rates, been it lower comparing to the Control. Day 6 embryos showed no difference in expansion and hatching for the vitrified groups, been them lower than the Control.

Discussion: Embryos obtained on day 6 are more sensible than the ones of day 5, fact observed when the embryos were exposed to cryoprotectant solution, as well by the behavior of the no treated Control embryos. The toxicity increases as it does the concentration of intracellular cryoprotectant, where over $16 \%$ of the intracellular cryoprotectors already affected the day 6 embryos development. For the day 5 embryos however, 15 or $16 \%$ of the intracellular cryoptrotectors, had similar behavior to the embryos. For the extracellular solutions, however, it is variable according the embryos development speed. Indeed, it is necessary to adjust the cryoprotectors to be used to cryopreserve porcine in vitro produced embryos obtained at days 5 and 6 of culture.
\end{abstract}

Keywords: porcine embryos, cryopreservation, parthenogenesis, cryoprotector, vitrification.

DOI: $10.22456 / 1679-9216.93774$ 


\section{INTRODUCTION}

Porcine embryos, especially the in vitro produced, are very sensible to the reproductive assisted manipulations. This impairs their viability, as it hinders the application of some reproductive tools, especially embryo cryopreservation, one of the only ways, up to date, for genetic storing.

Cryopreservation aids to preserve genetic material, as well as to disseminate it among different countries. Porcine embryos has already been successfully cryopreserved by vitrification $[3,5,6,9,10,13]$. The high cryoprotectant concentrations are responsible for providing cell protection, but its concentration and exposure time are critical points for survival. For instance, most protocols proposed for porcine embryo vitrification, when standardised using in vivo produced embryos, do not yield the same efficiency for the ones in vitro.

As it happens in vivo, during the in vitro development of porcine embryos, within the same batch, their growth can happen under different speeds. The embryos with best ability usually show faster development; therefore, embryo cryotolerance can be affected by their growing speed. We have hypothesized that fastest developing embryos would have better competence and higher cryotolerance.

This study aimed to assess the effect of different cryoprotectants combined in different vitrification protocols, in the toxicity and survival, for blastocysts of days 5 or 6 of culture.

\section{MATERIALS AND METHODS}

All chemicals were purchased from SigmaAldrich $^{1}$, unless otherwise noted.

\section{Experimental design}

Three experiments were conducted in order to access the cryoprotectants toxicity and embryos cryotolerance of days 5 and 6 parthenotes porcine embryos. In the first experiment, three different cryoprotectants were used (Dimethyl sulfoxide - DMSO, Ethylene glycol - EG, and Sucrose - SUC), combined in three different associations: G1: equilibration solution of $7.5 \% \mathrm{EG}+7.5 \% \mathrm{EG}$ for 5-15 min, and vitrification solution with $15 \% \mathrm{EG}+$ $15 \% \mathrm{DMSO}+0.5 \mathrm{M} \mathrm{SUC}$, warming was with $1 \mathrm{M}$ SUC for $1 \mathrm{~min}$ followed by $0.5 \mathrm{M}$ SUC for $3 \mathrm{~min}$; G2: equilibration solution of $7.5 \% \mathrm{EG}+7.5 \% \mathrm{EG}$ for $3 \mathrm{~min}$, and vitrification solution with $16 \% \mathrm{EG}+16 \% \mathrm{DMSO}$ with
0.4 M SUC for 1 min, warming was with 0.13 M SUC for $5 \mathrm{~min}$; G3: equilibration solution of $10 \% \mathrm{EG}+10 \%$ EG for 3 min and vitrification solution of $18 \% \mathrm{EG}+18 \%$ DMSO with $0.5 \mathrm{M}$ SUC for $1 \mathrm{~min}$, warming was with $1 \mathrm{M} \mathrm{SUC}$ for $1 \mathrm{~min}$ followed by $0.5 \mathrm{M} \mathrm{SUC}$ for $3 \mathrm{~min}$.

The second experiment compared EG combined to one of these three extracellular cryoprotectants: Polyvinylpyrrolidone/sucrose/trehalose (respectively groups: PVP, SUC, TRE). The equilibration solution was with $2 \mathrm{M}$ EG for $5 \mathrm{~min}$, and vitrification solution with $8 \mathrm{M}$ EG associated with: 7\% PVP-10 (group PVP), 0.4 M SUC (group SUC), or 0.6 M TRE (group TRE) in incubation for $1 \mathrm{~min}$. Warming of all the groups were done with $0.13 \mathrm{M} \mathrm{SUC}$ for $5 \mathrm{~min}$.

The third experiment evaluated four vitrification protocols for embryos of day 5: Prot 1- Equilibration solution with $7.5 \% \mathrm{EG}+7.5 \% \mathrm{DMSO}$ incubated for 5-15 min, vitrification solution with $15 \% \mathrm{EG}+15 \%$ DMSO + 0.5 M SUC incubated for $1 \mathrm{~min}$, warmed with $0.13 \mathrm{M}$ SUC for $5 \mathrm{~min}$; Prot 2- Equilibration solution with $7.5 \% \mathrm{EG}+7.5 \%$ DMSO incubated for 5-15 min, vitrification solution with $15 \% \mathrm{EG}+15 \% \mathrm{DMSO}+$ $0.5 \mathrm{M}$ SUC incubated for $1 \mathrm{~min}$, warmed with $0.13 \mathrm{M}$ TRE for $5 \mathrm{~min}$; Prot 3- Equilibration solution with 2 M EG 5 min, vitrification solution with $8 \mathrm{M} \mathrm{EG}+0.5$ M SUC for $1 \mathrm{~min}$, warmed with $0.13 \mathrm{M} \mathrm{SUC} \mathrm{for} 5$ min; Prot 4- Equilibration solution with $2 \mathrm{M} \mathrm{EG} 5 \mathrm{~min}$, vitrification solution with $8 \mathrm{M} \mathrm{EG}+0.5 \mathrm{M}$ SUC for $1 \mathrm{~min}$, warmed with $0.13 \mathrm{M}$ TRE for $5 \mathrm{~min}$; and four protocols for embryos of day 6: Prot 1- Equilibration solution with $7.5 \% \mathrm{EG}+7.5 \% \mathrm{DMSO}$ for $5-15 \mathrm{~min}$, vitrification solution with $15 \% \mathrm{EG}+15 \% \mathrm{DMSO}+0.6$ M TRE for $1 \mathrm{~min}$, warmed with $0.13 \mathrm{M} \mathrm{SAC}$ for $5 \mathrm{~min}$; Prot 2- Equilibration solution with 7.5\% EG $+7.5 \%$ DMSO for 5-15 min, vitrification solution with $15 \%$ $\mathrm{EG}+15 \% \mathrm{DMSO}+0.6 \mathrm{M}$ TRE for $1 \mathrm{~min}$, warmed with $0.13 \mathrm{M}$ TREA for $5 \mathrm{~min}$; Prot 3- Equilibration solution with $2 \mathrm{M}$ EG for $5 \mathrm{~min}$, vitrification solution with $8 \mathrm{M} \mathrm{EG}+0.6 \mathrm{M}$ TRE for $1 \mathrm{~min}$, warmed with $0.13 \mathrm{M}$ SUC for $5 \mathrm{~min}$; Prot 4- Equilibration solution with $2 \mathrm{M}$ EG for $5 \mathrm{~min}$, vitrification solution with 8 $\mathrm{M} \mathrm{EG}+0.6 \mathrm{M}$ TRE for $1 \mathrm{~min}$, warmed with $0.13 \mathrm{M}$ TREA for $5 \mathrm{~min}$.

For the three experiments, a group of fresh embryos was kept as Control.

\section{Oocytes collection and in vitro maturation (IVM)}

Gilt ovaries were transported from the abattoir to the laboratory where follicles measuring 3-6 Mm 
in diameter were aspirated with the aid of a vacuum pump connected to an $18 \mathrm{G}$ needle.

After morphological classification (under stereomicroscope in centrifuged follicular fluid), the cumulus oophorous complexes (COCs) considered of good or excellent quality grade were selected (completely surrounded by a compact layer of cumulus cells, with none or very discrete cytoplasmic granulation), according to previously described [2].

Selected COCs were submitted to in vitro maturation (IVM) into 4-well dishes ${ }^{2}$ containing $400 \mu \mathrm{L}$ of maturation medium, in groups of 40-50 COCs per well. During the first $22 \mathrm{~h}$ of IVM, the medium consisted in: TCM-199 $+25 \%(\mathrm{v} / \mathrm{v})$ of porcine follicular fluid $(\mathrm{PFF})+0.1 \mathrm{mg} / \mathrm{mL}$ cysteine $+10 \mathrm{ng} / \mathrm{mL}$ epidermal growth factor ${ }^{3}+0.91 \mathrm{mM}$ of sodium pyruvate $+3.05 \mathrm{mM}$ D-glucose $+0.5 \mathrm{mg} / \mathrm{mL}$ of luteinizing hormone (LH - Lutropin $)^{4}+0.01 \mathrm{UI} /$ $\mathrm{mL}$ follicle stimulating hormone (FSH - Folltropin) ${ }^{4}$ $+1 \mathrm{mM}$ of dibutiryl-cyclic-AMP (dbcAMP) +100 $\mathrm{IU} / \mathrm{mL}$ of penicilin $\mathrm{G}+0.1 \mathrm{mg} / \mathrm{mL}$ of streptomycin sulphate. An additional 20-24 $\mathrm{h}$ IVM period had the medium depleted of LH, FSH and the dbcAMP. All incubations were performed at $38.5^{\circ} \mathrm{C}$, in a $5 \% \mathrm{CO}_{2}$ atmosphere in air, and saturated humidity.

\section{Parthenogenetic activation and in vitro culture (IVC)}

The IVM oocytes were pipetted in manipulation medium (TCM-Hepes $+10 \%$ fetal calf serum - FCS) in order to remove their cumulus cells. Activation was through exposure to $15 \mu \mathrm{M}$ of ionomycin (in TCM-Hepes) for $5 \mathrm{~min}$, followed by a $4 \mathrm{~h}$ incubation in $2 \mathrm{mM}$ of 6-dimethylaminopurine in PZM-3 medium [15]. Activated oocytes were transferred to the in vitro culture (IVC) steps, and the onset of IVC was considered as day 0 .

The presumptive zygotes were cultured in medium PZM-3 under the same conditions as described for the IVM. On day 4, the medium was supplemented by adding $10 \%$ of FCS. Development to blastocyst was assessed on days 5, 6, 7 and 8 of IVC, according to the experiment.

\section{Embryo toxicity to cryoprotectants}

We tested different combinations of either intracellular (EG and DMSO), and extracellular cryoprotectants (SUC, TRE and PVP) dissolved in manipulation medium. Only blastocysts and expanded blastocysts obtained at days 5 and 6 of culture were used, being all of quality grade excellent (homogeneous cytoplasm without vacuoles or fragmentation) or good (homogeneous cytoplasm, with few vacuoles or fragmentation). The cryoprotectant concentrations and exposure times has been described previously in literature. This step of toxicity test was performed by exposing the embryos to equilibrium and vitrification solutions, followed by exposure to warming solutions, without plunging the embryos into the liquid nitrogen. Evaluations were performed up to day 8 .

\section{Vitrification of blastocysts}

Only good or excellent blastocysts and expanded blastocysts of days 5 and 6 were vitrified, in groups of 3-5. Embryos were exposed to an equilibrium solution (with intra-cellular cryoprotectant), and then placed into the vitrification solution (with intra- and extracellular cryoprotectants). Embryos were loaded into an open glass pulled micropipette, by capillarity, and plunged into LN2, according to each treatment, as described in the experimental design.

For the warming steps, the micropipette tips with the embryos were quickly immersed into warming solution, being rinsed in manipulation medium for additional $10 \mathrm{~min}$, and maintained in the IVC for further assessments.

Viability of blastocysts after toxicity tests and vitrification

Viability criteria were expansion (after $24 \mathrm{~h}$ ) and hatching rates (assessed every $24 \mathrm{~h}$ until day 8 ). At day 8 , all hatched and live non-hatched blastocysts were fixed in PBS $+4 \%$ formalin $+3 \%$ BSA, for assessment of the total cell number (TCN) and expression of apoptosis (by the expression of cleaved caspase-3 (CC3).

\section{Expression of cleaved caspase-3 (CC3) and blastocysts total cell number (TCN)}

The expression of CC3 indicates the programmed cell death. To assess that, embryos were fixed in $4 \%$ formalin $+3 \%$ BSA in PBS, for at least $20 \mathrm{~min}$, and incubated in PBS $+3 \% \mathrm{BSA}$ and $0.5 \%$ Triton X-100 (permeabilization solution) at $37^{\circ} \mathrm{C}$, for $1 \mathrm{~h}$. After a $2 \mathrm{x}$ rinse in $\mathrm{PBS}+0.2 \%$ tween $-20+$ $3 \%$ BSA (blocking solution), embryos were overnight exposed to the primary antibody anti-cleaved caspase-3 (1:400) in rabbit, and diluted in blocking solution. Embryos were then washed $3 x$ (20 min each) in blocking solution, incubated (for $2 \mathrm{~h}$ ) at $37^{\circ} \mathrm{C}$, in 
secondary antibody goat anti-rabbit Alexa Fluor 488 (1:400). Then, after one rinse (for $20 \mathrm{~min}$ ) in blocking solution, and another (20 $\mathrm{min}$ ) rinse in DAPI (1:1000) in blocking solution, embryos were mounted between slide and coverslip in a droplet of mowiol, to be observed under an inverted epi-fluorescent microscope (Olympus IX51) $)^{5}$.

The incidence of apoptosis in each embryo was estimated by the difference between the cells expressing CC3 (stained by Alexa Fluor 488) and the total cell count (stained by DAPI).

\section{Statistical analysis}

Data were analyzed using the JMP (software version 5,2002$)^{6}$. The proportion of positive cells for CC3 and the percentages of blastocysts expansion and hatching were analysed by Chi-square. The TCN values were submitted to ANOVA, being normalized, when necessary, by the arc sin of the square root of the data to be compared by the Tukey test. To all the experiments, the significance level was $5 \%$.

\section{RESULTS}

Experiment 1: Toxicity test for EG, DMSO and SUC in blastocysts of days 5 or 6 of culture

The Control of day 5 showed higher $(P \leq$ $0.05)$ expansion $(100.0 \%)$ and hatching $(60.8 \%)$ rates, compared to the treated groups. The groups G1 and G2 showed similar expansion rates (respectively 50.0 and $52.8 \%)$ between them, both higher than G3 (35.6\%). The hatching rates for treated day 5 embryos were similar amongst all treated embryos, respectively 15.3, 9.7 and $5.5 \%$ for G1, G2 and G3 (Table 1).

For day 6 embryos, the expansion rate for the Control (84.5\%) was higher than all treated groups, as follows: G1 (50.0\%) higher than G2 (31.0\%) and G3 (3.6\%), and G2 higher than G3. The hatching rate for the Control (39.7\%) was similar to G1 (23.2\%), being them both higher than G2 $(8.6 \%)$. The group G3 has the lowest hatching rate $(P \leq 0.05)[0.0 \%]$.

Expansion rates of day 6 Control embryos was $(84.5 \%)$ lower $(P<0.05)$ than for day 5 embryos $(100.0 \%)$ [Table 1]. When G2 and G3 were compared between days 5 and 6 , the expansion rates were higher for day 5.

Table 1. Viability of parthenote porcine embryos exposed or not to toxicity tests using EG, DMSO and SUC for blastocysts of days 5 and 6 of culture.

\begin{tabular}{|c|c|c|c|c|c|c|}
\hline \multirow[b]{2}{*}{ Group } & \multicolumn{3}{|c|}{ Embryos of day 5 of culture } & \multicolumn{3}{|c|}{ Embryos of day 6 of culture } \\
\hline & $\begin{array}{c}\text { Blastocysts } \\
\mathrm{n}\end{array}$ & $\begin{array}{c}\text { Expanded } \\
\mathrm{n}(\%)\end{array}$ & $\begin{array}{c}\text { Hatched } \\
\text { n }(\%)\end{array}$ & $\begin{array}{c}\text { Blastocysts } \\
\mathrm{n}\end{array}$ & $\begin{array}{c}\text { Expanded } \\
\mathrm{n}(\%)\end{array}$ & $\begin{array}{c}\text { Hatched } \\
\text { n }(\%)\end{array}$ \\
\hline Control & 74 & $74(100.0)^{\mathrm{aA}}$ & $45(60.8)^{\mathrm{aX}}$ & 58 & $49(84.5)^{\mathrm{aB}}$ & $23(39.7)^{\mathrm{a} Y}$ \\
\hline G1 & 72 & $36(50.0)^{\mathrm{bc}}$ & $11(15.3)^{b}$ & 56 & $28(50.0)^{\mathrm{b}}$ & $13(23.2)^{\mathrm{a}}$ \\
\hline G2 & 72 & $38(52.8)^{\mathrm{bA}}$ & $7(9.7)^{b}$ & 58 & $18(31.0)^{\mathrm{cB}}$ & $5(8.6)^{b}$ \\
\hline G3 & 73 & $26(35.6)^{\mathrm{cA}}$ & $4(5.5)^{\mathrm{b}}$ & 56 & $2(3.6)^{\mathrm{dB}}$ & $0(0.0)^{\mathrm{c}}$ \\
\hline
\end{tabular}

G1: Vitrification solution with 15\% EG + 15\% DMSO + 0.5 M SUC, warmed with 1 M SUC for 1 min followed by 3 min in 0.5 M SUC. G2: Vitrification solution with $16 \% \mathrm{EG}+16 \% \mathrm{DMSO}+0.4 \mathrm{M} \mathrm{SUC}$, warmed with $0.13 \mathrm{M}$ SUC for 5 min. G3: Vitrification solution with $18 \% \mathrm{EG}+18 \%$ DMSO + 0.5 M SUC, warmed with $1 \mathrm{M} \mathrm{SUC}$ for $1 \mathrm{~min}$ followed by $3 \mathrm{~min}$ in $0.5 \mathrm{M} \mathrm{SUC}$. ${ }^{\mathrm{a}, \mathrm{b}}$ Distinct letters in the same column shown difference $(P<0.05)$. ${ }^{\mathrm{A}, \mathrm{B}}$ Distinct letters in the same row shown difference in expanded blastocysts rates $(P<0.05)$. ${ }^{\mathrm{X}, \mathrm{Y}}$ Distinct letters in the same row shown difference in hatched blastocysts rates $(P<0.05)$.

The amount of total cells in day 5 embryos was higher for G1 (94.0 \pm 17.0$)$ than for G3 (45.8 \pm 12.6$)$, without significant differences amongst other groups (Table 2). Conversely, for day 6 embryos, the Control and G1 TCN were similar between them and higher than G2 (respectively 75.0 $\pm 3.6 ; 65.0 \pm 4.2$; and 33.0 \pm 6.2 ). The live non-hatched embryos of days 5 and 6 had similar TCN at day 8, despite the treatment.
For all treatments of both days 5 and 6, there was a higher proportion of CC3 in the non-hatched, in comparison to the hatched blastocysts (Table 2). Within the non-hatched, the higher incidence of CC3 was at day 6; whereas within the hatched, no differences were observed in the incidence of $\mathrm{CC} 3$, despite the treatment (including controls) for either day 5 and 6 (Table 2). 
Table 2. Total cell number (TCN) and percentage of cells expressing cleaved caspase-3 (CC3) at day 8 in blastocysts and expanded blastocysts after been exposed tor not to toxicity test at days 5 or 6 of culture.

\begin{tabular}{|c|c|c|c|c|c|}
\hline \multirow{2}{*}{ Group } & \multirow{2}{*}{ Toxicity test } & \multicolumn{2}{|c|}{ Not hatched embryos } & \multicolumn{2}{|c|}{ Hatched embryos } \\
\hline & & $\mathrm{TCN} \pm \mathrm{SE}$ & $\mathrm{CC} 3 \%$ & $\mathrm{TCN} \pm \mathrm{SE}$ & $\mathrm{CC} 3 \%$ \\
\hline Control & \multirow{4}{*}{ D5 } & $43.3 \pm 8.1$ & $21.5^{\mathrm{x}}$ & $75.3 \pm 7.1^{\mathrm{ab}}$ & $7.4^{\mathrm{Y}}$ \\
\hline G1 & & $34.0 \pm 9.8$ & $22.5^{\mathrm{x}}$ & $94.0 \pm 17.0^{\mathrm{a}}$ & $7.7^{\mathrm{Y}}$ \\
\hline $\mathrm{G} 2$ & & $49.5 \pm 12.2$ & $21.0^{X^{*}}$ & $67.7 \pm 12.2^{\mathrm{ab}^{*}}$ & $10.3^{\mathrm{Y}}$ \\
\hline G3 & & $36.0 \pm 25.3$ & $24.0^{x}$ & $45.8 \pm 12.6^{\mathrm{b}}$ & $9.7^{\mathrm{Y}}$ \\
\hline Control & \multirow{4}{*}{ D6 } & $39.6 \pm 8.4$ & $20.2^{\mathrm{Xy}}$ & $75.0 \pm 3.6^{x}$ & $8.1^{Y}$ \\
\hline G1 & & $35.0 \pm 10.8$ & $30.5^{x x}$ & $65.0 \pm 4.2^{\mathrm{x}}$ & $10.1^{\mathrm{Y}}$ \\
\hline $\mathrm{G} 2$ & & $39.8 \pm 9.4$ & $31.4^{\mathrm{Xx}^{*}}$ & $33.0 \pm 6.2^{y^{*}}$ & $6.1^{\mathrm{Y}}$ \\
\hline G3 & & $34.8 \pm 9.8$ & $30.5^{x}$ & 0.0 & 0.0 \\
\hline
\end{tabular}

G1: Vitrification solution with 15\% EG + 15\% DMSO + 0.5 M SUC, warmed with 1 M SUC for 1 min followed by 3 min in 0.5 M SUC. G2: Vitrification solution with $16 \% \mathrm{EG}+16 \% \mathrm{DMSO}+0.4 \mathrm{M} \mathrm{SUC}$, warmed with $0.13 \mathrm{M}$ SUC for 5 min. G3: Vitrification solution with $18 \%$ EG $+18 \% \mathrm{DMSO}+0.5 \mathrm{M} \mathrm{SUC}$, warmed with $1 \mathrm{M} \mathrm{SUC}$ for $1 \mathrm{~min}$ followed by $3 \mathrm{~min}$ in $0.5 \mathrm{M} \mathrm{SUC}$. ${ }^{\mathrm{X}, \mathrm{Y}}$ Distinct letters in the same row shown difference for CC3 $(P<0.05)$. ${ }^{\mathrm{a}, \mathrm{b}}$ Distinct letters in the same column shown difference for the TCN for embryos of day $5(P<0.05)$. ${ }^{\mathrm{x}, \mathrm{y}}$ Distinct letters in the same column shown difference for the TCN for embryos of day $6(P<0.05)$. *In the same column show difference between embryos of day 5 and 6 for the same group $(P<0.05)$.

Experiment 2: The effect of extracellular cryoprotectants: $P V P$, SUC and TRE

Under our conditions, PVP and SUC (68.7 vs $65.9 \%$ ) for day 5 embryos provided the best expansion rates in comparison to TRE (43.8\%), and all lower than the Control (89.9\%). The hatching rates were similar between SUC (28.0\%) and PVP (15.7\%), but higher than TRE (12.5\%), whilst PVP and TRE were similar between them (Table 3).

For day 6, TRE (58.5\%) kept an expansion rate similar to the Control (73.2\%). By the other hand, the group SAC (42.6\%) was similar to TRE and to PVP (39.3\%), been PVP lower than TRE and Control. For day 6 embryos, hatching was similar among Control (12.5\%), SUC (5.6\%) and TRE (5.5\%), being PVP (1.8\%) similar to SUC and TRE but lower than the Control (Table 3).
Experiment 3: Post-warming survival of porcine embryos, vitrified using different combinations of intra- and extracellular cryoprotectant agents

For day 5 embryos, the Fresh Controls showed the highest expansion and hatching rates. Amongst only the vitrified protocols, the expansion rate $(20.0 \%)$ of protoco1 (P1) was higher than all the other vitrified counterparts: P2, P3 and P4 (respectively $4.3 \%, 4.3 \%$ e $4.4 \%$ ). For the vitrified ones, the hatching rates were similar amongst them all (Table 4).

For day 6 embryos, the expansion and hatching rates after vitrification had no significant difference, although all they were significantly lower than the Fresh Control (Table 5).

Table 3. Porcine parthenotes embryos of days 5 and 6 exposed or not to toxicity test to the extracellular cryoprotectors PVP, SUC and TRE.

\begin{tabular}{|c|c|c|c|c|c|c|}
\hline \multirow[b]{2}{*}{ Group } & \multicolumn{3}{|c|}{ D5 } & \multicolumn{3}{|c|}{ D6 } \\
\hline & $\begin{array}{c}\text { Blastocysts } \\
\mathrm{n} \\
\end{array}$ & $\begin{array}{c}\text { Expanded } \\
\mathrm{n}(\%)\end{array}$ & $\begin{array}{c}\text { Hatched } \\
\mathrm{n}(\%)\end{array}$ & $\begin{array}{c}\text { Blastocysts } \\
\mathrm{n}(\%)\end{array}$ & $\begin{array}{c}\text { Expanded } \\
\mathrm{n}(\%)\end{array}$ & $\begin{array}{c}\text { Hatched } \\
\mathrm{n}(\%)\end{array}$ \\
\hline Control & 79 & $71(89.9)^{\mathrm{aA}}$ & $41(51.9)^{\mathrm{aX}}$ & 56 & $41(73.2)^{\mathrm{aB}}$ & $7(12.5)^{\mathrm{aZ}}$ \\
\hline PVP & 83 & $57(68.7)^{\mathrm{bA}}$ & $13(15.7)^{\mathrm{bcX}}$ & 56 & $22(39.3)^{\mathrm{cB}}$ & $1(1.8)^{\mathrm{bZ}}$ \\
\hline SAC & 82 & $54(65.9)^{\mathrm{bA}}$ & $23(28.0)^{\mathrm{bX}}$ & 54 & $23(42.6)^{\mathrm{bcB}}$ & $3(5.6)^{\mathrm{abZ}}$ \\
\hline TRE & 80 & $35(43.8)^{\mathrm{c}}$ & $10(12.5)^{\mathrm{c}}$ & 58 & $34(58.6)^{\mathrm{ab}}$ & $3(5.2)^{\mathrm{ab}}$ \\
\hline
\end{tabular}

a,b,c,Distinct letters in the same column shown difference $(P \leq 0.05){ }^{\mathrm{A}, \mathrm{B}}$ Distinct letters in the same row shown difference for the expansion rates $(P$ $\leq 0.05)$. ${ }^{\mathrm{X}, \mathrm{Z}}$ Distinct letters in the same row shown difference for the hatching rates $(P \leq 0.05)$. 
Table 4. Day five porcine parthenotes embryos viability after vitrification in four different protocols.

\begin{tabular}{cccc}
\hline \multirow{2}{*}{ Group } & \multicolumn{2}{c}{ D5 } \\
\cline { 2 - 4 } & Blastocysts & Expanded & Hatched \\
$\mathrm{n}(\%)$ & $\mathrm{n}(\%)$ \\
\hline Control & 46 & $44(95.7)^{\mathrm{a}}$ & $19(41.3)^{\mathrm{a}}$ \\
Prot 1 & 45 & $9(20.0)^{\mathrm{b}}$ & $1(2.2)^{\mathrm{b}}$ \\
Prot 2 & 47 & $2(4.3)^{\mathrm{c}}$ & $0(0.0)^{\mathrm{b}}$ \\
Prot 3 & 46 & $2(4.3)^{\mathrm{c}}$ & $0(0.0)^{\mathrm{b}}$ \\
Prot 4 & 45 & $2(4.4)^{\mathrm{c}}$ & $0(0.0)^{\mathrm{b}}$ \\
\hline
\end{tabular}

Prot 1: Equilibration solution with 7.5\% EG + 7.5\% DMSO incubated for 5-15 min, vitrification solution with 15\% EG + 15\% DMSO + 0.5 M SUC incubated for $1 \mathrm{~min}$, warmed with $0.13 \mathrm{M} \mathrm{SUC}$ for $5 \mathrm{~min}$. Prot 2: Equilibration solution with 7.5\% EG + 7.5\% DMSO incubated for 5-15 min, vitrification solution with 15\% EG + 15\% DMSO + 0.5 M SUC incubated for 1 min, warmed with $0.13 \mathrm{M}$ TRE for 5 min. Prot 3: Equilibration solution with 2 M EG 5 min, vitrification solution with 8 M EG + 0.5 M SUC for 1 min, warmed with 0.13 M SUC for 5 min. Prot 4: Equilibration solution with 2 M EG 5 min, vitrification solution with 8 M EG + 0.5 M SUC for 1 min, warmed with 0.13 M TRE for 5 min. a,b,c Distinct letters in the same column shown difference $(P \leq 0.05)$.

Table 5. Day six porcine parthenotes embryos viability after vitrification in four different protocols

\begin{tabular}{|c|c|c|c|}
\hline \multirow{3}{*}{ Group } & \multicolumn{3}{|c|}{ D6 } \\
\hline & Blastocysts & Expanded & Hatched \\
\hline & $\mathrm{n}$ & $\mathrm{n}(\%)$ & $\mathrm{n}(\%)$ \\
\hline Control & 60 & $52(86.7)^{\mathrm{a}}$ & $23(38.3)^{\mathrm{a}}$ \\
\hline Prot 1 & 62 & $7(11.3)^{b}$ & $0(0.0)^{\mathrm{b}}$ \\
\hline Prot 2 & 58 & $2(3.4)^{b}$ & $0(0.0)^{b}$ \\
\hline Prot 3 & 60 & $8(13.3)^{b}$ & $0(0.0)^{b}$ \\
\hline Prot 4 & 60 & $5(8.3)^{b}$ & $0(0.0)^{\mathrm{b}}$ \\
\hline \multicolumn{4}{|c|}{ 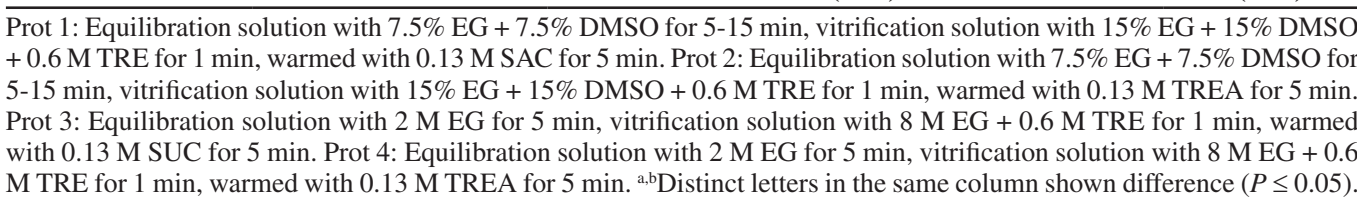 } \\
\hline
\end{tabular}

\section{DISCUSSION}

In order to provide an adequate cell cryoprotection, the concentration of cryprotectant agents shall be the most effective but safe, to avoid any toxic effect to the cells. In the porcine embryos it was found that $15 \%$ of intracellular cryoprotectant might not be enough to protect the cells [4]. Nevertheless, under our conditions, this concentration provided (for the toxicity tests) the highest expansion and hatching rates for the most sensible (day 6) embryos, and $16 \%$ already damaged the embryos. One possible explanation for the best results might be the higher concentration of SUC $(0.5 \mathrm{M})$ in comparison to what they $(0.4 \mathrm{M})$ used; even though it is a slight difference, the potential to dehydrate the cells can be importantly affected. These results confront what the same authors found when the association of $17 \%$ of intracellular cryoprotectants impaired the cell proliferation capacity of in vivo produced embryos; this treatment is equivalent to our experimental group G3, which consisted of $18 \%$ of each intracellular cryoprotectant.

The expression of $\mathrm{CC} 3$ is a reliable indicator of programmed cell death. According to results of the toxicity tests combining EG, DMSO and SUC, the embryos showing ability to hatch also presented similar cell viability, since they had similar patterns of CC3 expression. In spite of the highest sensibility of day 6 blastocysts and expanded blastocysts, it was possible to observe a higher incidence of $\mathrm{CC} 3$ expression in the embryos exposed to cryoprotectants, in comparison to their Controls, when all nonhatched (but morphologically alive) counterparts 
were analysed. This difference was not observed when day 5 embryos were assessed for CC3, what can be attributed to their better resistance, compared to day 6.

The use of EG as the only intracellular cryoprotectant for porcine embryo has been reported [1,4], often combined with some extracellular cryoprotectant, in steps of vitrification or warming. Between the extracellular cryoprotectants PVP [1] and SUC [4], the warming steps are more commonly held by SUC, during warming steps.

The use of TRE has provided very encouraging results, in spite of its known properties, such as the capacity to stabilize cell membranes under stressful conditions [14], or even its specific interactions with membrane phospholipids [7]. However, when combined as a cryoprotectant in our system, the expansion rate obtained was not above the satisfactory, not playing any positive role in the embryo hatching rates.

Our results also disagree from what Morató et al. [12] found, where day 6 blastocysts had not only better quality, but also higher survival (expansion) rates when compared to day 5 counterparts. By the other hand, embryos of day 6 are more sensible to specific culture medium [11]. As evidenced by the clear difference in sensitivity showed by embryos that reach the blastocyst stage under different developing speeds (at days 5 or 6 ), there is the need for directing specific vitrification protocols for either developing kinetics; the production system, howsoever, must be considered when one intends to select the best cryopreservation protocol.

\section{CONCLUSIONS}

In conclusion, day 5 embryos are more viable and resistant to cryoprotectors than day 6 embryos. Notwithstanding, the intracellular cryoprotectors EG and DMSO when used together are less toxic to embryos of day 5 of culture when used in a concentration up to $16 \%$. However, for embryos of day 6 , this limit goes up to $15 \%$ of each cryoprotector, whereas $16 \%$ already increases caspase in no hatched embryos, and reduces TCN of the hatched ones.

Besides the extracellular cryoprotectors showed a slight difference on its cell toxicity, the cryotolerance of the less toxic extracellular agents was not effective to promote embryo hatching.

\section{MANUFACTURERS}

${ }^{1}$ Sigma Chemical Co. St. Louis, MO, USA.

${ }^{2}$ SPL Lifescience Inc. Eumhyeon, South Korrea.

${ }^{3}$ Invitrogen Brasil Ltda. São Paulo, SP, Brazil.

${ }^{4}$ Bioniche Animal Health. Belleville, ON, Canada.

${ }^{5}$ Olympus Corporation. Tokyo, Japan.

${ }^{6}$ SAS Institute Inc. Cary, NC, USA.

Acknowledgements. The authors thank CAPES, CNPQ and FAPESC for the financial support.

Declaration of interest. The authors report no conflicts of interest. The authors alone are responsible for the content and writing of this manuscript.

\section{REFERENCES}

1 Beebe L.F.S., Cameron R.D.A., Blackshaw A.W. \& Keates H.L. 2005. Changes to porcine blastocyst vitrification methods and improved litter size after transfer. Theriogenology. 64: 879-890.

2 Brogni C.F., Ohlweiler L.U., Klein N., Mezzalira J.C., Cristani J. \& Mezzalira A. 2016. Pré-incubação dos espermatozoides suínos diminui polispermia e aumenta a produção embrionária em oócitos de baixa qualidade. Ciencia Rural. 46(6): 1113-1118.

3 Cameron R., Beebe L., Blackshaw A., Higgins A. \& Nottle M. 2000. Piglets born from vitrified early blastocysts using a simple technique. Australian Veterinary Journal. 78: 195-206.

4 Cuello C., Sanchez-Osorio J., Almiñana C., Gil M.A., Perals M.L., Lucas X., Roca J., Vazquez J.M. \& Martinez E.A. 2008. Effect of the cryoprotectant concentration on the in vitro embryo development and cell proliferation of OPS vitrified porcine blastocysts. Cryobiology. 56: 189-194.

5 Dobrinsky J. 1997. Cryopreservation of pig embryos. Journal of Reproduction and Fertility. (Suppl). 52: 301-312.

6 Du Y., Zhang T., Li J., Kragh P.M., Kuwayama M., Ieda S., Zhang X., Schmidt M., Bøgh I.B., Purup S., Pedersen A.M., Villemoes K., Yang H., Bolund L. \& Vajta G. 2007. Simplified cryopreservation of porcine cloned blastocysts. Cryobiology. 54: 181-187.

7 Hu J.H., Li Q.W., Li G., Jiang Z.L., Bu S.H., Yang H. \& Wang L.Q. 2009. The cryoprotective effect of trehalose supplementation on boar spermatozoa quality. Animal Reproduction Science. 112: 107-118. 
8 Li R., Liu Y., Pedersen H.S., Kragh P.M. \& Callesen H. 2013. Development and quality of porcine parthenogenetically activated embryos after removal of zona pellucida. Theriogenology. 80: 58-64.

9 Martinez E.A., Martinez C.A., Nohalez A., Sanchez-Osorio J., Vazquez J.M., Roca J., Parrilla I., Gil M.A. \& Cuello C. 2015. Nonsurgical deep uterine transfer of vitrified, in vivo-derived, porcine embryos is as effective as the default surgical approach. Scientific Reports. 5: 10587.

10 Men H., Zhao C., Si W., Murphy C.N., Spate L., Liu Y., Walters E.M., Samuel M.S., Prather R.S. \& Critser J.K. 2011. Birth of piglets from in vitro-produced, zona-intact porcine embryos vitrified in a closed system. Theriogenology. 76: 280-289.

11 Mito T., Yoshioka K., Noguchi M., Yamashita S., Misumi K., Hoshi T. \& Hoshi H. 2015. Birth of piglets from in vitro-produced porcine blastocysts vitrified and warmed in a chemically defined medium. Theriogenology. 84(8): 1314-1320.

12 Morató R., Castillo-Martin M., Yeste M. \& Bonet S. 2014. Cryotolerance of porcine in vitro-produced blastocysts relies on blastocyst stage and length of in vitro culture prior to vitrification. Reproduction Fertility and Development. 28(7): 886-892.

13 Nagashima H., Kashiwazaki N., Ashman R.J., Grupen C.G. \& Nottle M.B. 1995. Cryopreservation of porcine embryos. Nature. 374: 416.

14 Sampedro J.G. \& Uribe S. 2004. Trehalose-enzyme interactions result in structure stabilization and activity inhibition. The role of viscosity. Molecular and Cellular Biochemistry. 256: 319-327.

15 Yoshioka K., Susuki C., Tanaka A., Anas I. \& Iwamura S. 2002. Birth of piglets derived from porcine zygotes cultured in a chemically defined medium. Biology of Reproduction. 66: 112-119. 